\title{
Perceived Realism of Crowd Behaviour with Social Forces
}

\author{
Stuart O'Connor, Fotis Liarokapis, Chrisina Jayne \\ Coventry University, Masaryk University, Coventry University \\ \{oconno13@uni.coventry.ac.uk, liarokap@fi.muni.cz, ab1527@coventry.ac.uk\}
}

\begin{abstract}
This paper investigates the development of an urban crowd simulation for the purposes of psychophysical experimentation. Whilst artificial intelligence (AI) is advancing to produce more concise and interesting crowd behaviours, the number or sophistication of the algorithms implemented within a system does not necessarily guarantee its perceptual realism. Human perception is highly subjective and does not always conform to the reality of the situation. Therefore it is important to consider this aspect when dealing with AI implementations within a crowd system aimed at humans. In this research an initial two-alternative forced choice $(2 A F C)$ with constant stimuli psychophysical experiment is presented. The purpose of the experiment is to assess whether human participants perceive crowd behaviour with a social forces model to be more realistic. Results from the experiment suggest that participants do consider crowd behaviour with social forces to be more realistic. This research could inform the development of crowd-based systems, especially those that consider viewer perception to be important, such as for example video games and other media.
\end{abstract}

Keywords--- Crowd Simulation, Psychophysics, Perception, Artificial Intelligence, Agent Behaviour, Virtual Environments.

\section{Introduction}

The process of simulating huge crowds of intelligent agents in real-time is a challenging task due to numerous different considerations, technical or otherwise [12]. When the element of human perception is also taken into account, then this task becomes even more complex. Crowd behaviour is typically simulated with agent artificial intelligence algorithms (AI). The resultant behaviour can differ drastically depending on the specific algorithms that are implemented within a crowd based system, e.g. different pathfinding algorithms can deliver different paths for the same goal. Whilst some may assume that utilising lots of the most sophisticated algorithms would lead to believable behaviour, this is not always the case [16].
In light of this some form of quantification is needed in order to assess the behaviour of agents within crowd simulations. Realism, in the context of a crowd simulation, is the degree of plausibility of the crowd behaviour. Perceived realism in particular is a type of realism that is centered on the perception of humans. This type of realism is highly important for crowd simulations with a purpose geared towards interaction with humans, e.g. video games, and as such can be utilised to determine the degree of success for crowd behaviour [21].

Previous research has already utilised perception as a tool for evaluating crowds [7]. This research suggests a novel perspective of utilising psychophysics when evaluating crowd simulation systems. Psychophysics refers to a broad range of psychology based experiment methodologies, which are used in many ways to evaluate human perception through varying the levels of a stimuli and measuring the response [8]. Such methods when considered with perceived realism in mind can be adapted to perceptually evaluate the crowd behaviour of agents within a simulation.

A specific behavioral feature is selected in order to conduct a forced choice psychophysical experiment to assess the perceived realism of a single aspect. The selected behavioral feature is the social forces model [9], an algorithm that has been implemented in many different crowd simulations. To this end an urban crowd simulation is developed to produce varied stimuli for different intensities of the different social forces. These stimuli are utilised under the psychophysics method for comparing the perceived realism between virtual scenes with various intensity of social forces present and scenes without social forces present.

This research aims to address these aspects in the specific scope of an urban crowd simulation when considering perceptual realism. Whilst there has been some research towards evaluating crowds [4][23], the use of psychophysical methods to assess perceptual realism is an area open for further research. Typically research towards crowds is focused on further developing algorithms [10][13] and applying current methods to specific scenarios [1][3][26][27], so furthering knowledge for evaluation methods of crowd behaviour will add to the field. 
The rest of this paper is structured as follows: Section 2 presents background and related research. The implementation of the urban crowd simulation is detailed in section 3. Section 4 outlines the social forces psychophysics experiment and analyses the initial results. Section 5 provides conclusions and the direction of future research.

\section{Background}

As part of this research there are three main topics of interest, the simulation of agent crowd behaviour, perceptual realism and psychophysical evaluation methods. All of these elements tie into the overall research goal and its viability. These aspects are discussed in the following subsections.

\subsection{Agent Behaviour}

The simulation of agent crowd behaviour is a widely researched and applied field [4], with multiple applications ranging from serious simulations with specialized purposes, such as evacuation in response to bioterrorism [26], to simulations that aim to preserve heritage using virtual methods [27] or even simulations for urban planning [3]. The implemented agent behaviour often depends on a simulation's purpose and the context in which the agents are placed. For example a simulation for emergencies and evacuations [1] requires agents that are as close to reality as possible and behave as such, where as for an entertainment based simulation, e.g. a video game, this is not necessarily the case and behaviour can be simplified to a degree [2] to improve perception.

AI algorithms dictate the intricacies of the agent behaviour. A typical set-up includes a decision-making system [15], pathfinding navigation [6], local steering mechanics [25] and some form of a perception system [22]. These systems would allow an agent to make basic decisions and navigate the environment. Whilst this gives key behaviour, these base algorithms can be extended to give more advanced behavioral features, such as social aspects or learning. The specific algorithms will depend on the simulation's purpose and on what is believed to best achieve the desired type of behaviour. This means that it can be unclear on which algorithms or behavioral features to implement. In addition often algorithms or features will have parameter space or some form of customisability to consider as well.

\subsection{Perceived Realism}

When assessing crowd behaviour, realism is perhaps the key element to consider. Typically with any crowd simulation you want some degree of realism, however again the type of realism required is highly dependent upon the simulations purpose. In this respect there are multiple definitions for realism and how it is applied. Two types of realism include virtual realism and perceived realism. Virtual realism in the context of crowd simulation refers to the degree that the behaviour matches that of the reality. This type would be important in computer simulations, such as evacuation procedures. Perceived realism on the other hand refers to the degree a human viewer perceives the behaviour to be realistic in the given context or scenario. This type is more important for simulations that are for entertainment or similar purposes. These two definitions have been applied in the context of crowd simulation, by assessing the crowd behaviour.

The main consideration for this research is perceived realism, in terms of the perceptual responses from human participants. It is focused on entertainment based crowds or video games. Many recent video games releases feature crowd technologies as a major aspect, typically games with an open-world setting. In particular, popular game series such as Assassin's Creed published by Ubisoft and Grand Theft Auto published by Rockstar employ crowd based AI. Crowds are a part of the games virtual environment and in order for the game world to be perceived as realistic it must react in a real manner [14]. Implementing believable behaviour for crowds in this medium allows greater immersion for the participant and thus the game can achieve its purpose and engage the player [17].

\subsection{Psychophysics}

To assess the perceived realism of agent crowd behaviour, an experimental methodology is required. "Psychophysics is commonly defined as the quantitative branch of the study of perception, examining the relations between observed stimuli and responses and the reasons for those relations [5]." Whilst psychophysics is a useful tool for quantifying the human psyche to a degree, it is used infrequently in related research. Quite a few instances use related perceptual evaluation methods but do not adhere to a specific psychophysical method [7][19][23][24]. There are some instances of its use, for example one such experiment judging the perceptual thresholds for the smooth animation of characters under some given conditions utilised psychophysical methods [16]. This research shows that perception can be measured using such types of methods.

Psychophysics encapsulates multiple methods [8] that have different advantages and disadvantages for a given experiment. Some of the most common types include constant stimuli, staircase and forced choice methods. Constant stimuli and staircase are both methods for presenting levels of stimuli, with the former being a classical method and the latter being adaptive. Constant stimuli present the intensities of stimuli in a random order, so as to prevent guessing, whereas staircase adapts the level of stimuli based on individual participant responses. Forced choice on the other hand refers to constraints put upon an experiment, so that participants must respond in a short space of time. This method provides more reactive results and all trials are answered. 
The main advantage of using psychophysics is that allows the calculation of the psychometric function, which is used to determine the perceptual thresholds.

\section{General Methodology}

To conduct research on the perceived realism of behavioral features with regards to agent crowd behaviour, a general three-stage methodology is employed. Utilising this methodology over multiple iterations builds a corpus of perceptual data. For this iteration social forces is identified. In a previous iteration, varying velocity was the identified behavioral feature [20]. The three stages incorporate analysis, synthesis and perception [7]:

- Analysis: Analyse real-world instances of crowd behaviour in order to indentify a specific behavioral feature.

- Synthesis: Synthesise an updated simulation by refining the current build and implement the newly identified behavioral feature.

- Perception: Conduct a psychophysical experiment to assess the perceived realism of the newly implemented behavioral feature.

\section{Urban Crowd Simulation}

The development of the urban crowd simulation aims primarily to create a platform that is extensible with regards to the behavioral features of its crowds. Specific behavioral features can be implemented in a manner to allow control over key parameters of the algorithms. It is highly important, as part of psychophysics is to vary the intensity of presented stimuli and then collect the responses. As such more than just the configuration that the developer considered realistic is needed, and this is made available through the parameter spaces of the algorithms. The development of the simulation is conducted using the $\mathrm{C}++$ programming language, along with the OpenGL graphics API.

\subsection{Crowd Systems}

A procedural approach is selected to generate the urban environment. This is achieved by utilising an open source toolkit [11]. The layout of the city is calculated using land generation rules and then the geometry is overlaid. The final output is a virtual city of approximately $100 \mathrm{~km}^{2}$. Throughout simulation the frame rate remains interactive at $60 \mathrm{fps}$, with approximately 1000 agents active at any given time step.

The main consideration for a crowd-based system is to populate the virtual environment with intelligent agents. As stated previously in section 2.1, a typical implementation consists of four key elements: decision making, pathfinding, steering and perception. They allow the basic operations of an agent to perceive, think and act [2]. Within this system each agent is an individual entity with its own variables and data structure. The agents are updated at each frame during runtime.
For the decision-making system, finite state machines are chosen, as they offer a simple yet extensible approach. Currently the agents select their destinations as part of a main path following state. Additional states with various transitions can be added as they become necessary for creating specific behaviour.

For high-level navigation or pathfinding, the $\mathrm{A}^{*}$ algorithm is selected for implementation as it is proven robust [6] and widely utilised in video games. To calculate a path connected nodes in the environment are used. The resulting output is a list of nodes that an agent can follow to its destination. The algorithm calculates a value for each node to determine its efficiency for being searched, see equation 1 .

$$
\mathrm{F}=\mathrm{g}+\mathrm{h},
$$

where " $F$ " is the calculated fitness value for a specific node, essentially an estimate for the cost of a path going through that node, "g" is the goal value, which is the cost from the starting node to this node and " $\mathrm{h}$ " is heuristic value or the cost from this node to the destination.

For local navigation a type of steering is utilised [25]. For the urban crowd simulation the crowd path following type of steering is implemented. This allows agents to follow the paths between nodes, whilst avoiding excessive clustering, sometimes seen in crowd simulations. Figure 1 shows an example the pathfollowing element of this steering rule.



Figure 1: Path-following steering as implemented by Craig Reynolds [25]

A simple radial approach is selected for the synthetic perception. This type of perception allows an agent to access the objects and the other agents within its local neighborhood. This type of perception is common with flocking and types of steering, as seen in figure 2 .

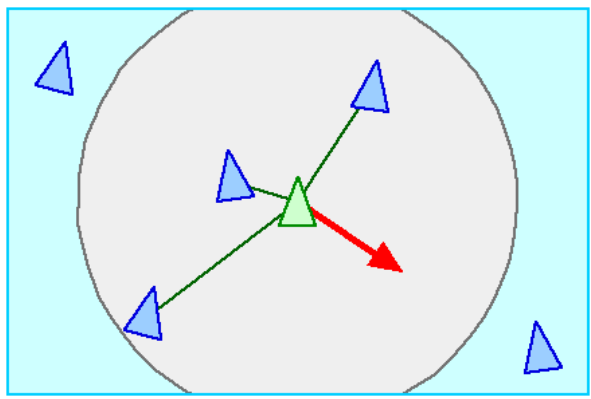

Figure 2: Radial perception utilised for a separation steering force [25] 
The behavioral features, in this case social forces, work in combination with the core systems to produce the agent behavior. See figure 3 .

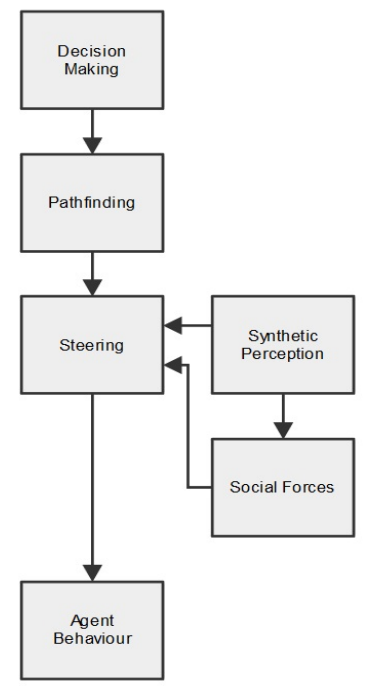

Figure 3: Diagram showing the linked nature between the main AI algoirthms and the agent behaviour.

\subsection{Social Forces}

Social forces can be used to describe the internal motivations or "social forces" of an agent to perform certain actions, in this case movement [9]. Typically a social forces model is implemented as several different forces performing different motions [18]. Commonly a model has a force towards a desired velocity, coupled with attractive and repulsive forces from other agents and objects. The final result of a social forces model is generally convincing behaviour, with the series of different forces leading to a style of self organisation that appear organic. Figure 4 highlights the agents and virtual environment at runtime.

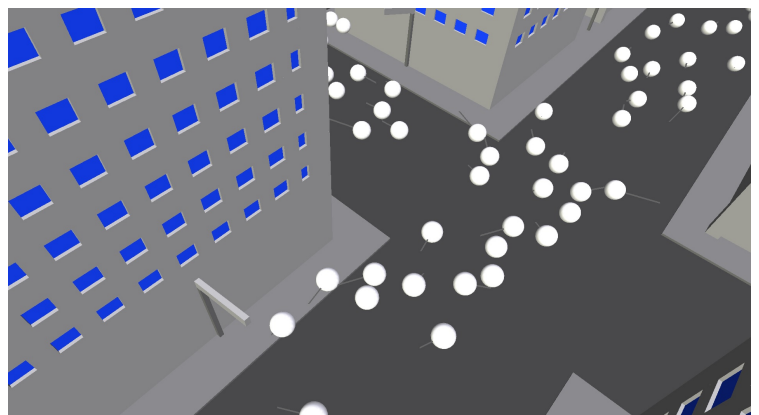

Figure 4: The simulation at runtime, with the four core $\mathrm{AI}$ algorithms and social forces in operation

For this urban crowd simulation, a social forces model is implemented using three specific forces for each agent: a repulsive force between agents, an attractive force between agents and a repulsive force from key objects. Each one of these is implemented with a weight factor as part of its parameter space, and thus the intensity of each force can be varied for the psychophysical experimentation. Equation 2 illustrates how the resultant steering force is calculated from the three forces present in the algorithm.

$$
\mathrm{SF}=(\mathrm{ar} * \mathrm{w})+(\mathrm{aa} * \mathrm{w})+(\text { or } * \mathrm{w}),
$$

where "SF" is the resultant calculated social force, which acts as a steering modifier. Agent repulsion is represented as "ar", agent attraction as "aa" and object repulsion as "or". Parameter weight is represented as the modifier "w". Calculated individually these forces are represented as shown in equations 3,4 and 5 .

$$
\begin{aligned}
& \text { ar }=\left(a-a^{n}\right) * n,(t, r) \\
& \text { aa }=\left(a^{n}-a\right) * n,(t, r) \\
& \text { or }=\left(a-o^{n}\right) * n,(t, r),
\end{aligned}
$$

where "a $\mathrm{a}^{\mathrm{n}}$ is the position of all the agents within vision and " $\mathrm{o}$ " is the position of all the objects within vision. "a" is the current agents position, " $n$ " is the normalizing factor, " $\mathrm{t}$ " is the time factor and " $\mathrm{f}$ " is the behaviour fluctuation factor.

Combining these three forces enables calculation of a social force that adds an additional steering vector to each agent. A good approximation of the social aspects of pedestrians can be achieved in this way. After implementation this behavioral feature is then utilised for perceptual experimentation, to discover if it adds to the perceived realism of the virtual crowd behaviour.

\section{Social Forces Psychophysics Experiment}

The next step is the perception stage, whereby a psychophysics-based experiment is conducted, in order to assess the perceptual realism of the newly implemented behavioral feature, namely social forces. As discussed in section 2.3, psychophysics consists of various methods and operations, each with advantages given certain scenarios. In this respect, a two-alternate forced choice (2AFC) constant stimuli approach is chosen consisting of both forced choice and constant stimuli.

The $2 \mathrm{AFC}$ element dictates the manner in which the stimuli are presented in the experiment. By being a forced choice experiment, the participant is given a limited amount of time and must make a choice regarding the presented stimuli. Constant stimuli means that the stimuli are presented in a random order to prevent participants from guessing any patterns. For each stimuli a perceived realism value is taken to determine if the participants finds that specific stimuli realistic or not.

\subsection{Experiment Setup}

By using an online survey platform specifically developed for the purposes of psychophysical experimentation, the order of the stimuli and responses can be tailored for each experiment. The platform is shown in figure 5. For this social forces experiment, the platform presents a number of trails to each participant. At each trail two videos are presented, one showing a specific intensity of a social force and the other not 
showing social forces implemented at all. At each trial the participants must respond to the presented stimuli and select which they perceive to show the most realistic crowd behaviour. They then assign it a percentage value for how perceptually realistic they feel it is.



Figure 5: A Trial of the online survey platform

The experiment consists of two key variables, one for each of the agent based social forces. These variables are tested at specific trials: trials 01 to 09 for agent avoidance and trials 10 to 18 for agent attraction. This accounts to a total of 18 trials. Using the initial parameter values derived from the real-world data during analysis, the values are then altered perceptually to produce the different stimuli intensities needed for the different trials. There are a total of nine different stimulus intensities per variable, at $10 \%$ intervals from $10 \%$ to $90 \%$.

\subsection{Results}

A total of 32 students participated in the social forces experiment, with 23 having a computing background. At each trial participants chose between a video with social forces and one without. They selected the one they believed to have the most realistic crowd behaviour. The results show that when social forces are implemented, participants perceive the crowd behaviour to be more realistic. A total of $94 \%$ of participants found that when the agent avoidance social force is present the behaviour of the agents is more realistic. $95 \%$ selected the videos with the agent attraction social force present to be more realistic. A total of $95 \%$ of the participants find a simulation with social forces to be more realistic.

The psychometric function is plotted in figure 6 . Using the perceived realism percentage values, it is possible to calculate the percentage seen value at each intensity. Figure 6 represents the percentage of participants that found social forces at that specific intensity, to produce reasonably realistic crowd behaviour. Perceived realism responses of $50 \%$ and over are considered as seen results and are represented in the psychometric function. Perceived realism responses below $50 \%$ or choices for the stimuli without social forces, are considered as unseen results. Only the two forces that are varied are represented, as the repulsive force from objects was kept constant over the trials.

By plotting the psychometric function using this data, the thresholds for the intensities of social forces that produce the most reliable perceptually realistic behaviour can be determined. Typically the thresholds

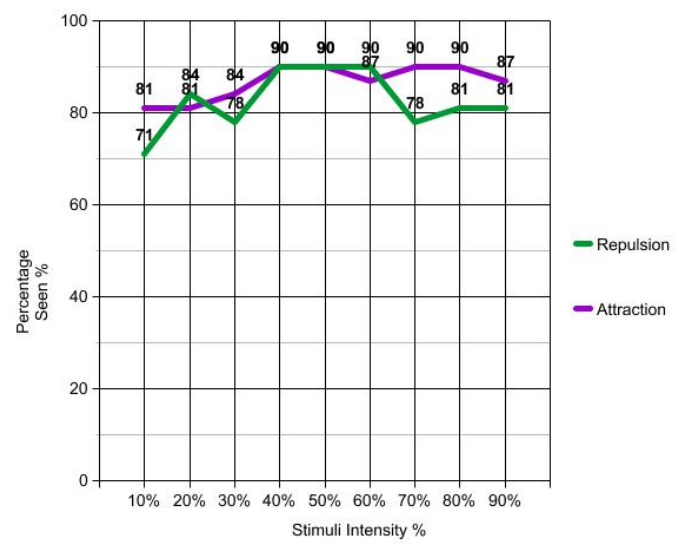

Figure 6: A graph showing the perceived realism responses as the psychometric function

are taken at the $75 \%$ seen mark, however the majority of the perceived realism responses are very high so the thresholds are mainly not visible. This means that the difference between the intensities could have been further increased to give a greater range of stimuli levels that highlight the threshold areas better. This is common for non-adaptive procedures where the intensity levels are probed, to improve the range for future experiments.

As the initial intensity at $50 \%$ is derived from realworld data, it would be a simple process to increase the gap between the different intensity levels. Figure 6 shows that the perceived realism values are at their highest seen when the stimuli intensities are between $40 \%$ and $60 \%$. This intensity range is closest to the initial intensity $50 \%$. For the attraction force there is also a peak at $70-80 \%$ intensity. At higher intensity levels this force causes agents to form denser groups more frequently. It is possible that this aspect could be perceived as more realistic and thus account for the slightly higher responses at higher intensities.

Certain elements of the simulations design may have contributed to the narrow range of responses. Agents are represented as basic geometry without animations. The changes in speed and direction between the different intensities could have been difficult to observe in this case. With pedestrian models and walking animations the behaviour may become more apparent and potentially lead to a greater distinction between intensity levels.

\section{Conclusions and Future work}

This paper shows results from experiments that utilise psychophysics to assess the perceived realism of agent crowd behaviour. The general methodology consists of analysing real world instances to identify behavioral features, that can be synthesised into the urban crowd simulation. Once complete perceptual experimentation is conducted so the behavioral features perceived realism is assessed, to determine its overall effectiveness for producing realistic crowd behaviour. The conducted research could inform the development of crowd-based systems and related perceptual experiments. 
In this specific instance the social forces behavioral feature is analysed and found to produce more perceptually plausibly crowd behaviour when compared to a simulation without social forces. By plotting the psychometric function, the range of stimuli is discovered to be too narrow. This range can be expanded for a future experiment. However it is possible that the use of a basic shape for pedestrians may have affected the final results, by making the small changes in agent velocity and direction between intensities potentially difficult to observe. Some intensities can still be seen to be slightly more effective than others. Overall it can be concluded that the impact of a social forces model on the perceived plausibility of agent crowd behaviour is considerable.

There are several avenues for future work including the further refinement of the current experiment with an updated stimuli range. This research can be also extended with identifying a new behavioral feature. In addition, the urban crowd simulation can be further developed through the use of games technology, to give access to additional features and allow for complex models and animations.

\section{References}

[1] Almeida, João E., Rosaldo JF Rosseti, and António Leça Coelho. "Crowd Simulation Modeling Applied to Emergency and Evacuation Simulations using MultiAgent Systems." arXiv preprint arXiv:1303.4692. 2013.

[2] Anderson, Eike F. "Playing smart-artificial intelligence in computer games." 2003.

[3] Aschwanden, Gideon, Jan Halatsch, and Gerhard Schmitt. "Crowd simulation for urban planning." In Architecture in Computro-26th eCAADe Conference Proceedings, pp. 493-500. 2008.

[4] Banerjee, Bikramjit, and Landon Kraemer. "Evaluation and comparison of multi-agent based crowd simulation systems." In Agents for games and simulations II, pp. 5366. Springer Berlin Heidelberg, 2011.

[5] Baird, John C, and Elliot Noma. "Fundamentals of Scaling and Psychophysics". John Wiley \& Sons, Inc. pp 1. 1978.

[6] Cui, Xiao, and Hao Shi. "A*-based pathfinding in modern computer games." International Journal of Computer Science and Network Security 11, no. 1, pp. 125-130. 2011.

[7] Ennis, Cathy, Christopher Peters, and Carol O'Sullivan. "Perceptual effects of scene context and viewpoint for virtual pedestrian crowds." ACM Transactions on Applied Perception (TAP) 8, no. 2, pp. 10. 2011.

[8] Ehrenstein, Walter H., and Addie Ehrenstein. "Psychophysical methods." In Modern techniques in neuroscience research, pp. 1211-1241. Springer Berlin Heidelberg, 1999.

[9] Helbing, Dirk, and Peter Molnar. "Social force model for pedestrian dynamics." Physical review E 51, no. 5, 4282. 1995.

[10] Kim, Sujeong, Stephen J. Guy, Dinesh Manocha, and Ming C. Lin. "Interactive simulation of dynamic crowd behaviors using general adaptation syndrome theory." ACM SIGGRAPH Symposium on Interactive $3 D$ Graphics and Games, pp. 55-62. ACM, 2012.

[11] Lance, Florien, Dimitri Matheossian, and Armand Poli. City Procedural Modelling Open Source Tool-kit,
GitHub, (Available at: https://github.com/Akado/Cityprocedural-modeling), Accessed at: 01/03/2013.

[12] Leggett, Richard. "Real-time crowd simulation: A review". R. Leggett, 2004.

[13] Lemercier, Samuel, A. Jelic, Richard Kulpa, Jiale Hua, Jérôme Fehrenbach, Pierre Degond, Cécile Appert-Rolland, Stéphane Donikian, and Julien Pettré. "Realistic following behaviors for crowd simulation." In Computer Graphics Forum, vol. 31, no.2 pt2, pp. 489498. Blackwell Publishing Ltd, 2012.

[14] Low, Gek Siong. "Understanding Realism in Computer Games through Phenomenology." 2001.

[15] Luo, Linbo, Suiping Zhou, Wentong Cai, Malcolm Yoke Hean Low, and Michael Lees. "Toward A Generic Framework for Modeling Human-like Behaviors in Crowd Simulation." 2009.

[16] McDonnell, Rachel, Fiona Newell, and Carol O'Sullivan. "Smooth movers: perceptually guided human motion simulation." In Proceedings of the 2007 ACM SIGGRAPH/Eurographics symposium on Computer animation, pp. 259-269. Eurographics Association, 2007.

[17] McMahan, Alison. "Immersion, engagement and presence." The video game theory reader, pp. 67-86. 2003.

[18] Mehran, Ramin, Alexis Oyama, and Mubarak Shah. "Abnormal crowd behavior detection using social force model." In Computer Vision and Pattern Recognition, 2009. CVPR 2009. IEEE Conference, pp. 935-942. 2009.

[19] Melo, Miguel, Maximino Bessa, Kurt Debattista, and Alan Chalmers. "Evaluation of HDR video tone mapping for mobile devices." Signal Processing: Image Communication 29, no. 2, pp. 247-256. 2014.

[20] O'Connor, Stuart, Fotis Liarokapis, and Christopher Peters. "An initial study to assess the perceived realism of agent crowd behaviour in a virtual city." In Games and Virtual Worlds for Serious Applications (VSGAMES), 2013 5th International Conference on, pp. 1-8. IEEE, 2013.

[21] O'Connor, Stuart, Fotis Liarokapis, and Christopher Peters. "A perceptual study into the behaviour of autonomous agents within a virtual urban environment." In World of Wireless, Mobile and Multimedia Networks (WoWMoM), 2013 IEEE 14th International Symposium and Workshops on a, pp. 1-6. IEEE, 2013.

[22] Ondřej, Jan, Julien Pettré, Anne-Hélène Olivier, and Stéphane Donikian. "A synthetic-vision based steering approach for crowd simulation." In ACM Transactions on Graphics (TOG), vol. 29, no. 4, p. 123. ACM, 2010.

[23] Peters, Christopher, and Cathy Ennis. "Modeling groups of plausible virtual pedestrians." IEEE Computer Graphics and Applications 4, pp. 54-63. 2009.

[24] Peters, Christopher, Cathy Ennis, Rachel McDonnell, and Carol O'Sullivan. "Crowds in context: Evaluating the perceptual plausibility of pedestrian orientations." Eurographics Short Papers, pp. 227-230. 2008.

[25] Reynolds, Craig W. "Steering behaviors for autonomous characters." In Game developers conference, vol. 1999, pp. 763-782. 1999.

[26] Song, Yiquan, Jianhua Gong, Yi Li, Tiejun Cui, Liqun Fang, and Wuchun Cao. "Crowd evacuation simulation for bioterrorism in micro-spatial environments based on virtual geographic environments." Safety science 53, pp. 105-113. 2013.

[27] Ulicny, Branislav, and Daniel Thalmann. "Crowd simulation for virtual heritage." In Proc. First International Workshop on 3D Virtual Heritage, no. VRLAB-CONF-2007-042, pp. 28-32. 2002. 